\title{
Flow-sensitive four-dimensional magnetic resonance imaging facilitates the quantitative analysis of systemic-to-pulmonary collateral flow in patients with univentricular hearts
}

\author{
Sarah Nordmeyer ${ }^{1 *}$, Israel Valverde ${ }^{2}$, Sergio Uribe ${ }^{3}$, Gerald F Greil ${ }^{2}$, Felix Berger ${ }^{1}$, Titus Kuehne ${ }^{1}$, \\ Philipp B Beerbaum²
}

From 15th Annual SCMR Scientific Sessions

Orlando, FL, USA. 2-5 February 2012

\section{Background}

Systemic-to-pulmonary collateral flow (SPCF) may constitute a risk factor for increased morbidity and mortality in patients with single-ventricle physiology (SV). However, clinical research is limited by the complexity of multi-site two-dimensional (2D) cardiovascular magnetic resonance (CMR) flow assessment. We sought to validate four-dimensional flow (4D-flow) for concise quantification of SPCF in patients with SV.

\section{Methods}

29 patients with SV physiology prospectively underwent CMR (1.5T) to quantify SPCF ( $\mathrm{n}=14$ bidirectional cavopulmonary connection [BCPC], age $2.9 \pm 1.3$ years; and $n=15$ Fontan, $14.4 \pm 5.9$ years) and 20 healthy volunteers (age, $28.7 \pm 13.1$ years) served as controls. Five $2 \mathrm{D}$-flow measurements (ascending aorta, superior/inferior caval veins, right/left pulmonary arteries) were performed and SPCF (=aortic minus caval flows) was calculated and compared with 4D-flow measurements and calculations. Additionally, 4D-flow measurements were used to calculate SPCF as pulmonary venous minus pulmonary arterial flow.

\section{Results}

The comparison between 4D-flow and 2D-flow showed good Bland-Altman agreement for all individual vessels (mean bias, $0.05 \pm 0.24 \mathrm{l} / \mathrm{min} / \mathrm{m} 2)$, calculated SPCF $(-0.02$ $\pm 0.18 \mathrm{l} / \mathrm{min} / \mathrm{m} 2)$, low intra and inter-observer variance

${ }^{1}$ Department of Congenital Heart Disease/Pediatric Cardiology, Deutsches Herzzentrum Berlin, Berlin, Germany

Full list of author information is available at the end of the article
(ICC >0.95[0.91-0.97]) and significantly shorter 4D-flow acquisition-time $(12: 34 \mathrm{~min} / 17: 28 \mathrm{~min}, \mathrm{p}<0.01)$. $4 \mathrm{D}$-flow in patients versus controls revealed (1) good agreement between systemic versus pulmonary estimator for SPFC; (2) significant SPCF in patients (BCPC $0.79 \pm 0.45 \mathrm{l} / \mathrm{min} /$ $\mathrm{m} 2$; Fontan $0.62 \pm 0.82 \mathrm{l} / \mathrm{min} / \mathrm{m} 2$ ) and not in controls $(0.01+0.16 \mathrm{l} / \mathrm{min} / \mathrm{m} 2)$ and (3) inverse relation of right/ left pulmonary artery perfusion and right/left SPCF (Pearson $=-0.47, \mathrm{p}=0.01$ ).

\section{Conclusions}

4D-flow is reliable, operator-independent and more timeefficient than 2D-flow to quantify SPCF. There is considerable SPCF in BCPC and Fontan patients. SPCF was more pronounced towards the respective lung with less pulmonary arterial flow suggesting more collateral flow where less anterograde branch pulmonary artery perfusion.

\section{Author details}

'Department of Congenital Heart Disease/Pediatric Cardiology, Deutsches Herzzentrum Berlin, Berlin, Germany. ${ }^{2}$ Pediatric Cardiology, King's College London, Division of Imaging Sciences, London, UK. ${ }^{3}$ Radiology and Biomedical Imaging Center, Pontificia Universidad Catolica de Chile, Santiago, Chile.

Published: 1 February 2012

doi:10.1186/1532-429X-14-S1-W7

Cite this article as: Nordmeyer et al.: Flow-sensitive four-dimensional magnetic resonance imaging facilitates the quantitative analysis of systemic-to-pulmonary collateral flow in patients with univentricular hearts. Journal of Cardiovascular Magnetic Resonance 2012 14(Suppl 1):W7.

\section{C) Biomed Central}

(c) 2012 Nordmeyer et al; licensee BioMed Central Ltd. This is an open access article distributed under the terms of the Creative Commons Attribution License (http://creativecommons.org/licenses/by/2.0), which permits unrestricted use, distribution, and reproduction in any medium, provided the original work is properly cited. 